\title{
$\rightarrow$ Götter, Gurus, Geist und Seele: HINDU-TRADITIONEN IN DER SCHWEIZ
}

\author{
MARTIN BAUMANN
}

\section{Einleitung}

Es ist nicht notwendig, an die Ufer des Ganges oder zu den heiligen Pilgerorten Indiens zu reisen, um hinduistische Feste und Tempelrituale mitzuerleben. Auch in der Schweiz finden sich Tempel, werden hinduistische Prozessionen durchgeführt und die Götter und Gurus mit grosser Hingabe verehrt. Ausserdem vermitteln einige der hiesigen Yoga-Schulen und -Zentren hinduistische Welt- und Wertvorstellungen. Der Yoga entstammt hinduistischen Übungsformen der Körperkontrolle mit dem Ziel der Kontrolle des Geistes. Im Westen wurde er im 20. Jahrhundert dann vielfach säkularisiert, indem die Praxis unabhängig von ihrem religiösen Bezug vermittelt und durchgeführt wurde.

Hinduismus in der Schweiz lässt sich in drei Gruppierungen unterteilen: 1. die Praktizierenden und Interessenten eines religionsunabhängigen Yoga sowie des religiös verstandenen Kriya-Yoga; 2. die Mitglieder und Sympathisierenden neohinduistischer Gruppen sowie 3. zugezogene Hindus aus Indien und Sri Lanka. Die einzelnen Gruppierungen wiederum sind vielgestaltig und oftmals mehr durch Unterschiede als durch Gemeinsamkeiten geprägt.

Der Beitrag skizziert in Teil 2 Grundzüge der Hindu-Traditionen und problematisiert die Kategorie »Hindu«. Teil 3 benennt die Anzahl von Hindus in der Schweiz, gefolgt von Teil 4 zu den unterschiedlichen hinduistischen Gruppierungen. Teil 5 schliesslich geht auf die Chancen und Risiken der Beziehungen zwischen hinduistischen Gruppierungen und schweizerischer Gesellschaft ein.

\section{Hindu-RELIGIONEN IN GRUNDZÜGEN}

\subsection{Vielfalt des Hinduismus}

Hinduismus in der Schweiz besteht aus einer Vielzahl unterschiedlicher Traditionen und Gruppen. Den Hinduismus und den Vertreter des Hinduismus gibt es in der Schweiz ebenso wenig wie in Indien und in anderen Ländern. Der Begriff »Hinduismus« ist eine britische Fremdbezeichnung des frühen 19. Jahrhunderts und bezeichnete die nichtchristlichen und nichtmuslimischen Personen auf dem indischen Subkontinent. Die Einheitlichkeit, die der Begriff unterstellt, existiert nicht. Es gibt keinen zentralen Text, Stifter oder Gott, und die Ritualpraktiken, Lehren, heili- 
gen Texte, religiösen Autoritäten und Organisationsformen der bis zu drei Jahrtausende alten Traditionen unterscheiden sich oftmals grundlegend. Einige Wissenschaftler schlugen daher vor, besser von »Hindu-Religionen « im Plural zu sprechen.

Dennoch lassen sich einige Gemeinsamkeiten benennen, so dass bei aller Unterschiedlichkeit von »Hinduismus« gesprochen werden kann. So ist zumindest in den meisten hinduistischen Traditionen die hingebungsvolle Verehrung der Götter und Göttinnen zentral. Die Götter gewähren Wünsche und Bitten, können aber auch Strafen und Unheil, Krankheit und Tod herbeiführen. Hindu-Sein bedeutet, den Göttern Respekt durch rituelle Handlungen zu erweisen, den Pflichten der Familie und Kaste nachzukommen und bestimmte religiöse Auffassungen zu teilen. Zu Letzteren gehören Vorstellungen von Reinheit/Unreinheit, Tatvergeltung (Karma), Seele, Wiedergeburt und der Möglichkeit, Befreiung aus dem anfangslosen Geburtenkreislauf erreichen zu können. Die Bhagavad-Gita, eine Versdichtung aus dem zweiten Jahrhundert vor unserer Zeitrechnung und bis heute eine der populärsten Schriften Indiens, beschreibt die Fortexistenz der Seele so: "Wie ein Mensch zerschlissene Kleider ablegt und neue, andere anlegt, so legt die Seele zerschlissene Körper ab, verbindet sich mit anderen, neuen« $(2,22)$.

\section{Hindu-Religionen}

Im gegenwärtigen, gelebten Hinduismus können drei Haupttraditionen als massgeblich gelten: Im Vishnuismus stehen der Gott Vishnu und seine irdischen Verkörperungen wie Krishna und Rama im Mittelpunkt der Verehrung. Diese Richtung ist besonders in Nordindien verbreitet, oft mit starker Betonung einer hingebungsvollen Gottesbeziehung.

Hindugläubige in der Tradition des Shivaismus verehren den Gott Shiva, mitunter auch als phallusartiger Stein (Lingam) symbolisiert. Bilder und figürliche Darstellungen zeigen ihn sowohl als Asketen im Himalaja als auch als Ehemann mit Frau und Söhnen. Shiva ist mächtig, kann zerstören, aber auch Gutes wirken. Ganesha, der elefantenköpfige ältere Sohn von Shiva, ist einer der beliebtesten Götter der Hindu-Traditionen. Er ist der Gott der Weisheit, der Überbringer von Glück und »Entferner der Hindernisse«. Zu Beginn jeder Puja (Andacht) wird er mit einer kleinen Opfergabe und einem Gebet bedacht. Murugan, der jüngere Sohn Shivas und Sinnbild für Schönheit, kriegerische Stärke und Schutzherr der tamilischen Sprache, wird besonders in Südindien und Sri Lanka verehrt. Im Shaktismus geniesst die Grosse Göttin höchsten Respekt. Sie tritt in vielen Formen und Namen auf, so als zerstörerische Kali oder 
ungestüme, siegreiche Durga. Zugleich ist sie auch wohlwollend und gütig, so in Darstellungen als Göttin Devi. Die Göttin verkörpert als Ehefrau Shivas die zerstörerische Seite seiner Macht und Kraft (Shakti).

Schätzungen gehen von weltweit 800 Millionen Hindus aus, etwa 95 Prozent leben im Ursprungsland Indien. Der Grossteil der Hindus in der Schweiz sind Verehrer und Verehrerinnen von Shiva und Shakti. Zugehörige zur International Society for Krishna Consciousness (ISKCON, »Hare Krishna«) verehren den Gott Krishna und weitere Götter der vishnuitischen Tradition. Der in Südindien lebende Guru Sathya Sai Baba (geb. 1926) wird sowohl von einigen Schweizer Konvertiten als auch indischen und tamilischen Hindus verehrt.

\subsection{Kategorisierung: Selbst- und Fremdzuschreibung}

Bei weitem nicht alle, die unter die obige Annäherung an den Hinduismus-Begriff fallen, verstehen sich als Hindus. Praktizierende der Transzendentalen Meditation betonen, sie seien an kein hinduistisches Weltverständnis gebunden und religiös neutral. Einige Yoga-Schulen können der Kategorie »Hindu « zugerechnet werden, viele nicht, obwohl sie die traditionellen Praktiken vermitteln. Es stellt sich die Frage, wie viele und welche Aspekte einen bzw. eine Hindu definieren. Der Kriya-Yoga fusst auf einem hinduistischen Weltbild, die Verehrung von Göttern und Rituale sind jedoch nebensächlich. Weiter: Lange Zeit, z.T. heute noch, lehnten orthodoxe Hindus die »neuen« westlichen, konvertierten Hindus ab. Ihrer Auffassung nach müsse ein Hindu in eine Kaste geboren sein; eine Konversion sei nicht möglich, da man Hindu nur durch Geburt sei. Schliesslich: Die Bezeichnung »Hindu« ist in Indien und Übersee vielen Gläubigen unverständlich, da sie sich als Verehrer Murugans oder Devis verstehen oder Teil einer bestimmten Gurutradition (Sanskrit: sampradaya) sind. Der Allgemeinbegriff Hindu taucht gar nicht auf. Die Grenzen der Einteilung sind fliessend und ermöglichen keine fixe Zurechnung, was im Folgenden zu beachten ist.

\section{Zahlen und Profil}

Hindu-Religionen in der Schweiz sind von den Zahlen her ein randständiges Phänomen. Die eidgenössische Volkszählung 2000 ermittelte 28.000 Personen, die auf dem Fragebogen ihre Zugehörigkeit mit »Hindu« bzw. »hinduistisch« angaben (o,4 \% der Bevölkerung). Die tatsächliche Zahl dürfte jedoch höher liegen, da die Antwortkategorie »hinduis- 
tische Gemeinschaft « nicht vorgegeben war und von der Selbstbezeichnung »Hindu« nicht ohne Weiteres auszugehen ist.

Der Grossteil hiesiger Hindus sind tamilische Flüchtlinge aus Sri Lanka, die in den 8oer und goer Jahren vor dem Bürgerkrieg flohen. Ende 2005 lebten knapp 42.000 Staatsbürger aus Sri Lanka, davon 7.300 Eingebürgerte, in der Schweiz. Der weit überwiegende Teil (90-95\%) von ihnen sind Tamilen, von diesen wiederum die meisten Hindus (80$85 \%$ ). Die Zahl tamilischer Hindus in der Schweiz dürfte demnach bei etwa 30.000 bis 34.000 Personen liegen. Zu diesen sind knapp 8.000 indische Hindus, geschätzte 4.000 bis 7.000 westliche, konvertierte Hindus sowie eine bislang nicht erhobene Zahl Yoga-Praktizierender (auf religiöser Grundlage) hinzuzuzählen. Insgesamt dürfte von 42.000 bis 50.000 Hindus (0,7 \% der Bevölkerung) für die Schweiz auszugehen sein.

Neun von zehn in der Volkszählung erfassten Hindus sind ausländischer Herkunft (92,5\%), lediglich 2.100 Schweizer und Schweizerinnen gaben an, einer hinduistischen Vereinigung zuzugehören. Wie die Auswertung der Volkszählung im Weiteren aufzeigt, ist das Ausbildungsniveau von Hindus deutlich unterdurchschnittlich: 65,5 Prozent verfügen über eine Ausbildung der Sekundarstufe I (Gesamtbevölkerung: 29,5 \%) und 17 Prozent über einen tertiären Abschluss (Gesamtbevölkerung: 19,2\%). In der jetzt heranwachsenden Generation dürfte sich dieses aufgrund des Bildungsehrgeizes jedoch deutlich zugunsten höherer Ausbildungsabschlüsse verschieben. Der Grossteil der Hindus ist bislang beruflich im Gastgewerbe (38,2\%) sowie im Gesundheits- und Sozialwesen (11,9\%) tätig. Ihr Aufenthaltsstatus als Flüchtling - einige sind seit 20 Jahren »vorläufig Aufgenommene« - zwingt sie zur Annahme jeglicher Arbeit, insbesondere in den Billiglohnbranchen. Der geringe Lohn ermöglicht jedoch nicht, einen gesicherten Bleibestatus (B-Bewilligung) und damit bessere Arbeitsoptionen zu erlangen. Der überwiegende Anteil der Hindus lebt, wie andere Migrantengruppen auch, in den Grossstädten, Schwerpunkte sind Bern und Zürich. Markant: Hindus weisen den landesweit höchsten Anteil endogamer Paare auf, d.h. Personen gleicher religiöser oder kultureller Zugehörigkeit heiraten untereinander. Zurückzuführen ist dieses auf die tamilische Praxis arrangierter Heiraten, die auch in der Diaspora Bestand hat. 


\section{Vielfalt hinduistischer TRAditionen UND GRUPPIERUNGEN IN DER SCHWEIZ}

\subsection{Interesse an Yoga}

Weithin wenig bekannt ist, dass der heute so populäre Yoga seine Wurzeln in den hinduistischen Traditionen hat. "Yoga " leitet sich aus dem Sanskrit her (der Verbwurzel » $y u j \lll)$ und kann mit »sich anstrengen, sich vorbereiten« übersetzt werden, ebenso mit »anspannen « - wie ein Rind in ein Joch gespannt wird. Im hinduistischen Weltverständnis geht es darum, durch körperliche Techniken den Geist »anzuspannen«, um ihn der Erleuchtung, der »Befreiung « aus dem Kreislauf von Wiedergeburten, zuzuführen. Die Körperstellungen, die so genannten Asana, sind Mittel, den Geist zu disziplinieren und das intuitive, nicht-rationale Erkennen und damit letztendlich Erleuchtung, Samadhi, zu ermöglichen.

In der Schweiz hat Yoga in den 8oer und goer Jahren des 20. Jahrhunderts ein grosses Interesse gefunden. Dieses zeigt sich an den zahlreichen Kursangeboten und Yoga-Schulen. Für die Gegenwart lassen sich die zwei Hauptströmungen des säkularisierten Yoga sowie des Kriya-Yoga, dem ein hinduistisches Menschen- und Weltbild zugrunde liegt, unterscheiden.

\subsubsection{Yoga als Entspannungsmethode}

Hinduistisches Gedankengut und Körperübungen des Yoga wurden in der Schweiz zu Beginn des 20. Jahrhunderts im Umfeld der Lebensreformbewegung bekannt. Diese zivilisationskritische Bewegung strebte durch eine Rückkehr zu als natürlich propagierten Lebens-, Ernährungsund Gemeinschaftsformen eine grundsätzliche Erneuerung der Gesellschaft und individuellen Lebensweise an. Auf dem Monte Verità, der bald berühmten »vegetarischen Kolonie« oberhalb von Ascona, übten sich Künstler, Esoteriker, Anarchisten, Theosophen und viele weitere in Stellungen des Yoga.

Dieses Interesse erhielt durch die Vorträge und das literarische Wirken des indischen Reformers Vivekananda (1863-1902) weiteren Auftrieb. Vivekanandas psychologisierende, in westliche Begriffe übertragene Neuinterpretation des Yoga für weltliche Ziele wurde zum wichtigsten Bezugspunkt aller nachfolgenden westlichen und indischen Neuinterpretationen des Yoga. Carl Gustav Jung (1875-1961), der grosse Schweizer Psychiater, deutete den Yoga psychologisch-therapeutisch. Er verwandte Yoga-Übungen - möglicherweise als erster Praktizierender in der Schweiz - zur Beruhigung und zur Kontrolle »aufwühlender Gefühle«. Jung und weitere Indienrezipienten begründeten die Grund- 
orientierung eines nichtreligiös fundierten Yoga: Nicht mehr die religiöse Zielrichtung - die Befreiung aus dem Geburtenkreislauf - war leitend, sondern Aspekte wie Körperertüchtigung, Stressbewältigung und Beruhigung mittels bewusst ausgeführter Körperübungen.

Erste Yoga-Gruppen entstanden nach dem Zweiten Weltkrieg Ende der $40 e r$ Jahre. Die Initialzündung ging von der Zürcher Yoga-Schule Yesudian-Haich aus. Selvarajan Yesudian (1916-1998) und die ungarische Künstlerin Elisabeth Haich (1897-1994) entwickelten ein pädagogisch und körperbetont ausgerichtetes Yoga - ihr Buch Yoga und Sport (1941) hob den körperstärkenden und gesundheitsfördernden Aspekt hervor. Sie eröffneten 1948 eine Yoga-Schule in Zürich und eine Sommerschule in Ponte Tresa im Tessin. In den $50 e r$ Jahren kamen Filialen in St. Gallen, Genf, Basel, Aarau und Luzern hinzu.

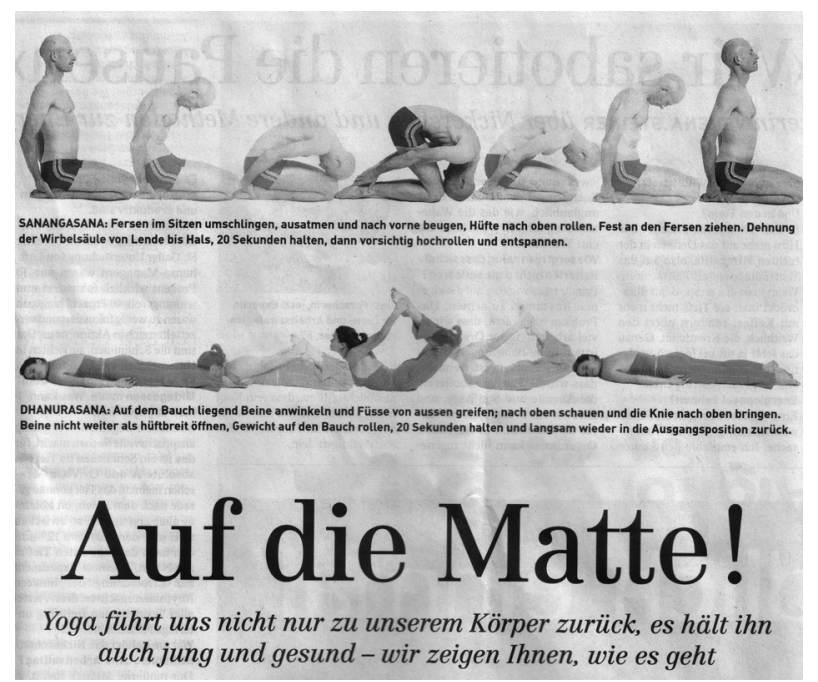

Abbildung 1: Illustration zum Zeitungsbeitrag über Yoga. Quelle: SonntagsZeitung, 16.10.2005, S. 125, Rubrik »Fit \& Well«, Autorin: Cornelia Heim

Den indisch-ungarischen Pionieren folgten ab den 6oer Jahren mit indischen Yoga-Lehrern, so etwa B.K.S. Iyengar, weitere ausländische und schweizerische Lehrer und Lehrerinnen. Organisatorisch erfolgte ein erster Zusammenschluss 1968 in der Schweizerischen Yoga Gesellschaft - ein Berufsverband von Yoga-Lehrern und -Lehrerinnen - sowie $1995 \mathrm{im}$ Schweizer Yogaverband. Letzterem sind etwa 350 Lehrer schweizweit angeschlossen (Stand: Sept. 2006), wobei einzelne bis zu 200 wöchentliche Teilnehmer verzeichnen. Beide Verbände, denen etwa 
die Hälfte der grob geschätzten 1.500 Yoga-Lehrer und -Lehrerinnen angehören, bieten Kurse vornehmlich in der Ausrichtung von Entspannungsmethoden, Körpererfahrungen, Atemübungen, Selbstfindung und »Harmonisierung" an. Mitunter sind auch »Power-Yoga«, Schwangerschafts- und Kinder-Yoga im Angebot. Journalistische Schätzungen gehen von 5 Prozent der Bevölkerung aus, die Yoga regelmässig praktizieren.

\subsubsection{Yoga als religiöse Praxis: Kriya-Yoga}

Die Übungen des Kriya-Yoga wurden in Nordamerika und Europa durch Paramahansa Yogananda (1893-1952), Gründer der Self Realization Fellowship 1925 in Los Angeles und Autor des legendären Buches Autobiographie eines Yogi (1946, deutsch 1950), bekannt. In den Folgejahren entstanden verschiedene Kriya-Yoga-Schulen und Ansätze, je mit eigenen, zumeist internationalen Organisationen. In der Schweiz ist seit den goer Jahren ein reges Interesse zu verzeichnen.

\section{Kriya-Yoga}

Kriya-Yoga beansprucht, eine Verbindung der verschiedenen Yoga-Arten darzustellen und legt den Übungen hinduistische Konzepte von Mensch, Seele und Kosmos zugrunde. Der bzw. die Praktizierende soll durch Atem-, Körper- und Konzentrationsübungen zu höheren Einsichten geführt werden und ein Verständnis entwickeln, dass die individuelle Seele (Atman) mit der allumfassenden Seele (Brahman) identisch ist. In Selbstdarstellungen wird der religiöse Bezug mitunter minimiert: »Kriya-Yoga ist [...] weder eine Sekte noch eine Religionsgemeinschaft. Vielmehr ist es ein Weg, die eigene wahre Identität wieder zu erlangen, unabhängig von Religion und Glaube.« (www.kriya-yoga.net)

Als erste Kriya-Yoga-Schule gründete die Self Realization Fellowship gemäss der eigenen Webseite 1952 in Zürich ein Zentrum. Mittlerweile finden sich zahlreiche Kriya-Yoga-Zentren von Schülern Yoganandas bzw. wiederum von deren Schülern. Einige seien genannt: Swami Paramahansa Hariharananda Giri (1907-2002), direkter Schüler Yoganandas, gründete in der Schweiz vier Kriya-Yoga-Zentren. Dessen Schüler Yogi Dhirananda (geb. 1937), seit 1997 mit Wohnsitz in Bregenz, rief 15 Kriya-Yoga-Gruppen ins Leben. Der Schüler von Yogi Dhirananda, Sri Janakananda (Schweizer), gründete etwa zehn »Yoga für alle«-Gruppen, mit Zentren in Anlehnung an indische Ashrams in Zofingen AG und in der Toscana.

Sowohl Kriya-Yoga als auch Yoga als Entspannungsmethode wer- 
den auffallend häufiger von Frauen als von Männern praktiziert. Die Frauen-Männer-Relation liegt bei etwa 80 zu 20 Prozent, sowohl bei Teilnehmern an Kursen als auch bei Yoga-Lehrerinnen und -Lehrern. Als wichtigste Gründe des grossen Interesses von Frauen an Yoga-Übungsformen sind körperbewusste, nicht leistungsbezogene Körperertüchtigung, Entspannungsmöglichkeiten angesichts der Doppelbelastung von Beruf und Haushalt sowie der Wunsch nach Selbsterfahrung und »/nnenschau« zu nennen.

\subsection{Neohinduistische Gruppen und Gemeinschaften}

In den 7oer und 8oer Jahren weckten neohinduistische Lehrer ein breites Interesse an hinduistischen Frömmigkeitsformen und Lehren. Die Herausbildung neuer hinduistischer Ansätze, oft unter dem Begriff Neohinduismus zusammengefasst, geht auf das 19. Jahrhundert und den Kontakt gelehrter Inder mit westlichen Ideen und britischen Missionaren zurück. Im Gegensatz zu orthodoxen Hindus betrachteten Reformer, allen voran Vivekananda, Hinduismus als universale Religion. Jedermann könne durch Übernahme hinduistischer Glaubensbezüge zum Hinduismus konvertieren.

Die 6oer Jahre des 20. Jahrhunderts bildeten den Beginn der Ausbreitung und Etablierung neohinduistischer Bewegungen in Nordamerika und Europa. Es war die Zeit, als religiöse Sucher, Hippies, Künstler und Morgenlandfahrer euphorisch nach Indien reisten, um dort »echte« Spiritualität und »ursprüngliche« Religiosität zu finden. Zugleich reisten indische Swamis (Gelehrte) und Gurus (Lehrer) in den Westen, um ihre Ideen zu verbreiten.

Als erster neohinduistischer Lehrer kam der indische Mönch Swami Omkarananda (1930-2000) in die Schweiz. Er rief 1966 das Divine Light Zentrum in Winterthur ins Leben und hatte in den Folgejahren aufgrund seiner Person und Betonung hinduistischer Spiritualität und Meditation regen Zulauf. Das Zentrum rief durch seine »Fremdheit« und seinen Zulauf Kritik in der Nachbarschaft hervor, was militante Gegenreaktionen der Zentrumsaktivisten zur Folge hatte; der Streit kam schnell in die Medien und eskalierte, als 1975 Anhänger ein Sprengstoffattentat auf das Haus eines Zürcher Regierungsrates verübten. Omkarananda wurde zu einer Haftstrafe und nachfolgender Landesverweisung verurteilt.

Ungleich ruhiger erfolgte 1969 die Gründung des Vedanta-Zentrums in Genf (Centre Védantique de Genève), das aus der 1962 ins Leben gerufenen Association pour l'étude du Védanta en Europe hervorgegangen war. Den Schwerpunkt der Aktivitäten bilden Studien der Lehre des Vedanta in der Deutung Vivekanandas und die Organisation von Ta- 
gungen. Vedanta bezeichnet die hinduistische Lehrauffassung der letztendlichen Einheit allen Seins. Dem Zentrum gehörten 2003 ca. 60 Mitglieder und ein Kreis von etwa 600 Interessenten an.

In den frühen 7oer Jahren traten mit den Gemeinschaften um Bhagwan Shree Rajneesh (1931-1990) und A.C. Bhaktivedanta Swami Prabhupada (1896-1977) zwei in der Öffentlichkeit stark, oftmals kontrovers wahrgenommene hinduistische Gurus hinzu. Die zwei charismatischen Lehrer sammelten rasch eine Anhängerschar spiritueller Sucher um sich. Die Neo-Sannyas-Bewegung um Bhagwan rief zahlreiche Ashrams und Zentren ins Leben, verlor nach dem Tod des »Meisters « 1990 jedoch an Zusammenhalt und Mitgliedern. Stabiler entwickelte sich ISKCON, die 1966 von Swami Prabhupada gegründete International Society for Krishna Consciousness, bekannt als Hare Krishna. Die in Orange gekleideten Mönche zogen singend durch Fussgängerzonen und erregten Erstaunen und Verwunderung. Der erste Krishna-Tempel in der Schweiz wurde 1972 in einem Vorort von Genf eröffnet, gefolgt vom Krishna-Zentrum 1975 in Zürich. Fünf Jahre später konnte am Zürichberg ein grossräumiges Haus erworben werden, wo seitdem der schweizerische Haupttempel besteht. Der Krishna-Gemeinschaft Schweiz dürften um die 400 Mitglieder und geschätzte 3.000 bis 5.000 Interessenten zuzurechnen sein. Interessant: Den Zürcher Krishna-Tempel besuchen seit den goer Jahren auch verstärkt tamilische Hindus aus Sri Lanka. Sie feiern mit tamilischen Landsleuten sonntags gemeinsam Andacht und lassen von den Mönchen für ihre Kinder lebensbegleitende Rituale durchführen.

Neben den genannten Gemeinschaften bestehen noch zahlreiche weitere neohinduistische Gruppen. Ananda Marga, der von Prabhat Ranjan Sarkar begründete "Weg zur höchsten Glückseligkeit«, kam 1972 in die Schweiz. Ihm folgte die Transzendentale Meditation (TM), die 1973 für ihren Gründer Maharishi Mahesh Yogi und zu Ausbildungszwecken ein ehemaliges Grand Hotel auf dem Seelisberg (Kanton Uri) erwarb. Die TM machte in den goer Jahren durch die Naturgesetzpartei und jüngst durch Zeitungsinserate zur Umgestaltung Schweizer Städte auf sich aufmerksam. Im Weiteren entstanden Gruppen um Sri Chinmoy, Sathya Sai Baba, Sri Babaji, Sri Sri Ravi Shankar (The Art of Living), Avatara Devi, Sri Hans Ji Maharaj (Divine Light Mission). In kleinen Gruppen vertreten sind Brahma Kumaris, Krishnamurti Foundation, Sahaja Yoga, Siddha Yoga, Shri Ram Chandra Mission.

Gegenwärtig sind die Gruppen und Gemeinschaften von der Anzahl ihrer Mitglieder und Interessenten her zumeist klein, im Bereich zwischen wenigen Personen (5-10 Mitglieder) bis zu 200 bis 300 Mitglie- 
dern. Insgesamt ist die Mitglieder- und Interessentenzahl in den vergangenen Jahren deutlich zurückgegangen.

\subsection{Hindu-Traditionen südasiatischer Zuwanderer}

\subsubsection{Indische Hindus}

Hindus aus Indien kamen als Studierende und gelernte Arbeiter seit den 7oer Jahren in die Schweiz. Sie sind eine an Medienberichten gemessen "stille« Migrantengruppe, die in der schweizerischen Öffentlichkeit bislang wenig bekannt ist. Die in allen grösseren Städten der Schweiz vorhandenen indischen Restaurants weisen am deutlichsten auf Indien und indische Kultur hin. Es existiert zwar eine Anzahl von lokalen Kultur- und Sprachvereinen. Einen eigenen Tempel mit regelmässigem kultischen Dienst eröffneten die ca. 8.000 indischen Hindus bislang jedoch nicht. Zum einen besuchen sie den von vielen indischen Hindus mit grossem Respekt angesehenen Krishna-Tempel in Zürich. Zum anderen leben die indischen Familien sehr verstreut über die Schweiz, ein Zusammengehörigkeitsgefühl besteht allenfalls sporadisch. Die »Indian Association Zürich« veranstaltet zu den Hindufesten Navaratri und Diwali (jeweils im Herbst) Feiern in grösserem Rahmen. Der Verein ist jedoch vorrangig indisch-kulturell und nicht spezifisch hinduistisch ausgerichtet.

\section{4-3.2 Tamilische Hindus aus Sri Lanka}

Tamilen verliessen Sri Lanka seit den späten 7oer Jahren, als der schon seit längerer Zeit schwelende Konflikt zwischen der singhalesischen Mehrheit und der tamilischen Minderheit eskalierte. Nach dem Pogrom 1983 stieg die Zahl tamilischer Flüchtlinge und Asylbewerber stark an. In den 8oer Jahren bestimmten Medienberichte über Kleinkriminalitätsund Drogendelikte von tamilischen Asylbewerbern das Bild in der Öffentlichkeit. Mit der zunehmenden ökonomischen und sozialen Eingliederung setzte in den goer Jahren ein merklicher Wandel in der öffentlichen Wahrnehmung ein. Nun wurden den tamilischen Flüchtlingen überwiegend positive Eigenschaften wie Fleiss, Zuverlässigkeit, Anpassungsfähigkeit und Lernbereitschaft zugeschrieben.

Die ökonomische und soziale Eingliederung der Tamilen ging jedoch nicht auf Kosten des Bewahrens religiöser und kultureller Traditionen. Tamilische Hindus richteten in einem Quartiertreffpunkt in Bern (1984), in einem Asylbewerberheim in Langnau BE (1984) und in einem Kellerraum in Basel (1986) erste kleine Tempel ein. In den goer Jahren, insbesondere in Folge des familiären Nachzugs von Frauen und Kindern, 
wurden weitere Tempel eröffnet, oft in umgewidmeten Lager- und Werkhallen. Sie bieten genügend Raum für die zahlreichen Götterschreine und Gläubigen. Im Herbst 2006 bestanden 17 Tempel, in denen die Götter und Göttinnen verehrt und die vielen Jahresfeste gefeiert werden. Einzelne Tempel führen zum Hauptjahresfest, dem zehntägigen Tempelfest, öffentliche Prozessionen um den Tempel herum durch, unter Beteiligung von 3.000 bis 4.000 tamilischen Besuchern. Hinduistische Religiosität findet jedoch ebenso zu Hause vor dem heimischen Schrein durch die Huldigung der durch Bilder und kleine Statuen dargestellten Götter statt.

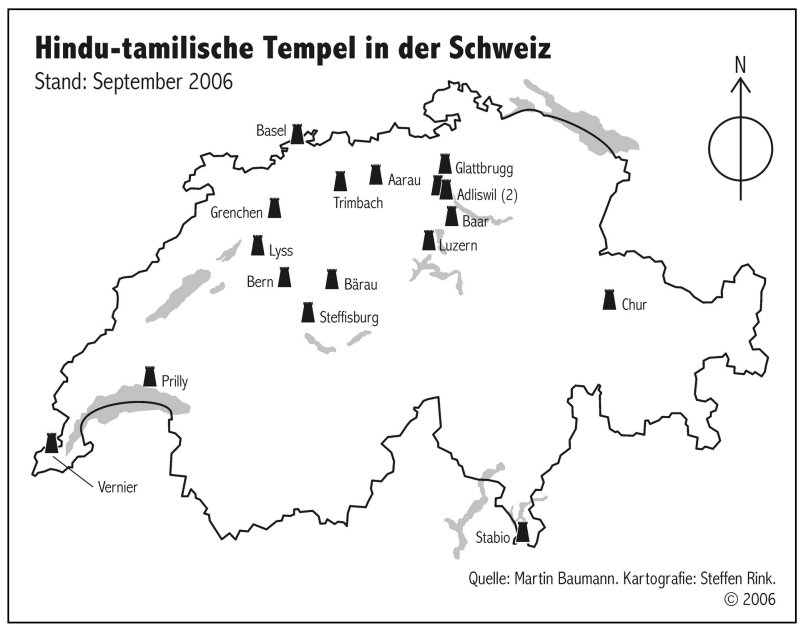

Abbildung 2: Hindu-tamilische Tempel in der Schweiz,

September 2006.

Quelle: (c) Martin Baumann, 2006

Die Tempel verfügen zumeist über gemauerte Schreine und sind den shivaitischen Göttern Murukan und Vinayakar (Ganesha) oder der Grossen Göttin (Thurkkai/Durga, Manonmani) geweiht. Die Tempel sind von aussen zumeist nicht als geweihte Orte der Götter erkennbar, innen findet sich jedoch eine südasiatische Welt en miniature. Mit der Eröffnung eines Tempels ist ein Abschotten und Rückzug in die eigene Gruppe nicht beabsichtigt. Hindu-Tempel tragen vielmehr zum Heimisch-Werden der Flüchtlinge und Zuwanderer bei. Für viele bilden diese Orte eine kleine Heimat in der Fremde, in der die vertraute Sprache gesprochen und gewohnte Umgangsformen gepflegt werden. Die sakralen Orte stärken die eigene Identität und Selbstvergewisserung.

Da Hindus glauben, dass sich das Göttliche in vielerlei Erscheinun- 
gen an besonderen Orten zeigt, besuchen viele hinduistische Tamilen überdies katholische Wallfahrtskirchen mit einer schwarzen Madonna.

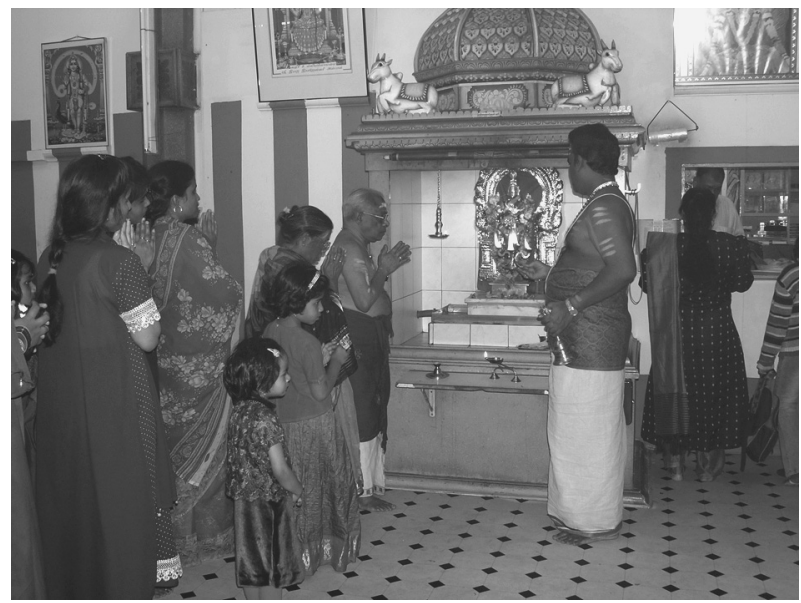

Abbildung 3: Puja (Andacht) im Sri Sivasubramaniar Tempel in Adliswil. Der Priester verehrt die Götter mit Licht (Flamme), Musik (Glocke) und Mantren (»kraftgeladene Silben«).

Quelle: (c) Martin Baumann, 2004

Dort bitten sie die Mutter Gottes - von ihnen als Grosse Göttin Devi, Ma oder Mata verehrt - um Schutz, Hilfe und Unterstützung. Besonders die Marienstätten Kloster Einsiedeln, Mariastein (bei Basel), St. Josef in Köniz (bei Bern) sowie Madonna del Sasso (bei Locarno) sind als »kraftspendende« und »reine Orte» beliebte Wallfahrtsziele hinduistischer (wie auch römisch-katholischer) Tamilen und Tamilinnen. Die hinduistische Verehrung der Maria ist dabei kein neues, diasporabedingtes Phänomen, sondern schon aus Sri Lanka und Südindien bekannt.

\section{Hinduismus in der SCHWeizerischen Gesellschaft}

Die Vielzahl hinduistischer Gruppierungen zeigt sich besonders im städtischen Umfeld. Jüngere Lokalstudien belegen dies: Im Kanton Luzern bestanden im Frühjahr 2005 sieben nichtreligiöse Yoga-Schulen bzw. -Kursanbieter, vier neohinduistische Gruppen sowie der hindu-tamilische Sri Thurkkai Amman Tempel. Wesentlich grösser zeigte sich die interne Pluralität im Grossraum Zürich, wo zirka eine Million Menschen leben: 2005 fanden sich ca. 35 nichtreligiöse Yoga-Kursanbieter, sechs Kriya-Yoga-Schulen, der Tempel der Krishna-Gemeinschaft, etwa 20 
neohinduistische Gruppen sowie drei hindu-tamilische Tempel in der Nähe Zürichs. Trotz der räumlichen Nähe stehen die verschiedenen Gruppen kaum oder gar nicht miteinander in Kontakt, zu gross sind die Unterschiede zwischen den verschiedenen Ausrichtungen.

Welche Chancen und Risiken beinhalten die Hindu-Traditionen für die Schweizer Gesellschaft? Die Vielzahl von Yoga-Schulen und YogaKursen, einerlei ob auf Entspannung oder religiöse Einsicht ausgerichtet, passt sich gewissermassen nahtlos in schweizerisches Leben ein. Gerade im städtischen Umfeld, in dem eindeutig die meisten Schulen und Zentren angesiedelt sind, ist es nicht mehr ungewöhnlich, sich als Übende bzw. Übender des Yoga zu erkennen zu geben. Yoga wird positiv als eine Form von Lifestyle und von Wellness, wenn auch der etwas anstrengenderen Art, angesehen. Sowohl im Kontext des Trends zu gesunder Lebensführung - hier durch körperliche Ertüchtigung - als auch im Zug des Esoterik- und Spiritualitätsbooms erfährt Yoga ein stark wachsendes Interesse. Gerade Letzteres deckt sich mit Motiven der Yoga-Aufnahme in lebensreformerischen Kreisen zu Beginn des 20. Jahrhunderts. War diese Rezeption jedoch ausdrücklich auch politisch und emanzipatorisch orientiert, so ist eine solche gesellschaftliche Dimension heutzutage nicht vorhanden. Die Perspektive zielt vielmehr auf die bzw. den Einzelnen, auf den eigenen Körper und auf körperliche, gefühlte Selbsterfahrung, um so bewusst von gesellschaftlichen Problemlagen fortzuführen. Auch hierin, dem zugeschriebenen Unpolitischen, liegt ein weiterer Grund der wachsenden Attraktivität.

Im Gegensatz dazu löste das öffentliche Auftreten neohinduistischer Gruppen in den 7oer und 8oer Jahren zum Teil vehemente gesellschaftliche Diskussionen aus. Der Fall um Swami Omkarananda eskalierte durch Überreaktionen auf beiden Seiten. Aufgrund einzelner Vorkommnisse klassifizierten christliche Weltanschauungsexperten neohinduistische Gruppen und weitere neue Religionen pauschal als »Sekten« und »ugendreligionen«, ohne die interne Vielgestaltigkeit und Unterschiedlichkeit zu berücksichtigen. Vorgeworfen wurde ihnen u.a. Freiheitsentzug und Mitglieder in die Abhängigkeit eines »Gurus« geführt zu haben. Die z.T. berechtigten Vorwürfe betrafen Einzelfälle, die jedoch nicht pauschal als typisch für alle neohinduistischen Gruppierungen dargestellt werden können. Mittlerweile, auch aufgrund interner Reformprozesse, ist es in der Öffentlichkeit weitgehend still um neohinduistische Gruppen geworden.

Mit Blick auf die grösste Hindu-Gruppe, die tamilischen Flüchtlinge aus Sri Lanka, ist festzuhalten, dass gesellschaftliche Konflikte im Bereich Religion/Hinduismus nicht auftraten. Kennzeichnend für die grosse Mehrheit tamilischer Zuwanderer ist eine starke Kompromissbereit- 
schaft und eine deutliche Zurückhaltung, Forderungen zu stellen. Auch aus diesem Grunde, neben den finanziellen, werden die Tempel in stadtfernen Industriegebieten errichtet. Möglichen Konflikten mit Nachbarschaften wegen etwaiger Ruhestörungen und Parkplatzproblemen wollen diese Gemeinschaften im Vornherein aus dem Wege gehen.

Im Unterschied zu den neohinduistischen Gruppen haben indische und tamilische Hindus ihre religiösen Aktivitäten eindeutig auf die eigenen Landsleute ausgerichtet. Missionarische Tendenzen sind bislang nicht vorhanden. Besucher und Besucherinnen in den Hindu-Tempeln sind willkommen. Tempelkomitees reagieren auf die zunehmenden Anfragen nach organisierten Besuchen und öffnen ihre Tempel für Schulklassen, Lehrerfortbildungskurse und weitere Interessentenkreise. Die Begegnungen zwischen nichttamilischen und tamilischen Bürgern in den Tempeln haben viele aufgrund der beobachteten tiefen Frömmigkeit der Hindus als bereichernde Erfahrung empfunden. Deutlich wird dabei oft auch, dass gelebte »andere « Religiosität eine gelingende gesellschaftliche Eingliederung nicht ausschliesst, eine Einsicht, die auf weitere hinduistische Gruppierungen wie auch auf andere Religionen übertragen werden kann.

\section{Vertiefende Literatur}

\subsection{Allgemein zum Hinduismus}

Knott, Kim (2000): Hinduismus. Eine kurze Einführung, Stuttgart: Reclam.

Michaels, Axel (1998): Der Hinduismus. Geschichte und Gegenwart, München: Beck.

\subsection{Hinduismus in der Schweiz}

Baier, Karl (1998): Yoga auf dem Weg nach Westen, Würzburg: Königshausen \& Neumann.

Baumann, Martin/Luchesi, Brigitte/Wilke, Annette (Hg.) (2003): Tempel und Tamilen in zweiter Heimat. Hindus aus Sri Lanka im deutschsprachigen und skandinavischen Raum, Würzburg: Ergon. Darin drei Kapitel zu tamilischen Hindus in der Schweiz.

Beltz, Johannes (2004): Hindu-ABC, Zürich: Präsidialdepartement der Stadt Zürich und Museum Rietberg.

Markus, Vera (2005): In der Heimat ihrer Kinder. Tamilen in der Schweiz, Zürich: Offizin. 
McDowell, Christopher (1996): A Tamil Asylum Diaspora. Sri Lankan Migration, Settlement and Politics in Switzerland, Oxford, Providence, RI: Berghahn Books.

Messerli, Barbara (1998): Maria wird zur Göttin. Hindu-TamilInnen verehren die christliche Madonna, Lizentiatsarbeit, Institut für Religionswissenschaft der Universität Bern.

Schneider, Alexandra (Hg.) (2002): Bollywood. Das indische Kino und die Schweiz. Zürich: Edition Museum für Gestaltung.

Vögeli, Johanna, 2004, Sumangali, die Glücksverheissende. Tamilisch-hinduistische Frauen in der Schweiz, Bern: Reformierte Kirchen Bern-Jura-Solothurn, Fachstelle Migration.

\subsection{Filmdokumente}

„Tempelfest des Sri Sivasubramania Tempels in Adliswil 2001«, von Willi Bühler mit Beratung von Eveline Masilamani-Meyer, SF 1 Fernsehbericht, Zürich, 60 min.

»Neujahrsfest und Tempelfest 2004 des Sri Thurkkai Amman Tempels in Root», von Kerstin-Katja Sindemann und dem Religionswissenschaftlichen Seminar der Universität Luzern, Luzern, 2X 15 min.

„Wanakam« von Thomas Isler, Zürich 2004, 82 min. Portrait von je zwei tamilischen Familien und Einzelpersonen in der Schweiz, denen die schlechte Bezahlung im Niedriglohnsektor eine Verstetigung ihres Aufenthalts nicht ermöglicht.

\subsection{Internet-Adressen (Primärquellen, Auswahl)}

Hindu Tempel Basel, siehe www.rel-news.ch/index.php?id=i1342

Krishna-Gemeinschaft Schweiz, Zürich: www.krishna.ch

Kriya-Yoga, Yogi Dhirananda: www.kriya-yoga.net

Schweizer Yogaverband: www.swissyoga.ch

Schweizerische Yoga Gesellschaft: www.yoga.ch

Sri Sivasubramania Tempel, in Adliswil: www.murugantemple-zh.ch

Sri Thurkkai Amman Tempel Luzern: http://durga.bhakthi.net, auch unter: www.religionenlu.ch/info-hindu1.html

Sri Vishnu Thurkkai Amman Tempel, in Adliswil: www.srithurkkaswiss.ch 